\title{
Polyphenol oxidase-based luminescent enzyme hydrogel: an efficient redox active immobilized scaffold
}

\author{
BISWAJIT DEY ${ }^{1, *}$, SUPRABHAT MUKHERJEE ${ }^{2}$, NILADRI MUKHERJEE ${ }^{2}$, \\ RANJAN KUMAR MONDAL ${ }^{1}$, BISWARUP SATPATI ${ }^{3}$ and SANTI PRASAD SINHA BABU ${ }^{2}$ \\ ${ }^{1}$ Department of Chemistry, Visva-Bharati University, Santiniketan 731235, India \\ ${ }^{2}$ Department of Zoology, Visva-Bharati University, Santiniketan 731235, India \\ ${ }^{3}$ Surface Physics and Material Science Division, Saha Institute of Nuclear Physics, 1/AF, Bidhannagar, Kolkata 700064 , \\ India \\ *Author for correspondence (bdeychem@gmail.com)
}

MS received 3 October 2016; accepted 6 July 2017; published online 2 February 2018

\begin{abstract}
A novel, functionally potent polyphenol oxidase (10000 U)-mediated urchin-shaped composite-based luminescent enzyme hydrogel network as immobilized scaffold for oxido-reductase efficiency on phenolic substrates including phenol, resorcinol, catechol and quinol was synthesized and characterized through fluorescence spectroscopy along with scanning electron microscopy and transmission electron microscopy.
\end{abstract}

Keywords. Polyphenol oxidase; enzyme hydrogel; microstructure; fluorescence; redox activity; phenolic substrates.

\section{Introduction}

Science esteems hydrogel [1,2] for its countless functionalities ranging from educational domain to industrial research [3-8]. Supramolecular hydrogel is an exclusively smart material originating through several non-covalent type interactions, including electrostatic interactions, hydrogen bonding interactions, aromatic ring-based interactions, van der Waals forces, hydrophobic interactions, etc. $[3,9,10]$. The structural flexibility of the supramolecular hydrogel network is advantageous to originate not only as potent nano-material, but also as some intriguing soft functional scaffold-like DNA-hydrogel $[9,11]$. The supramolecular hydrogel shows a numerous number of bio-applications like diagnosis and therapy, involving bioimaging, biodetection and tissue engineering [10]. So, the research on supramolecular hydrogel material is growing day by day to offer novel functional materials. Encapsulation of enzyme into the hydrogel networks [12-14] is an extraordinary research technique for the enzyme immobilization that immensely triggers the uses of enzyme in a controlled way to have reusable enzyme activity. There is a little number of literature reports on indirect enzyme-based hydrogel formation strategy $[15,16]$.

Polyphenol oxidase (PPO) is an industrially important enzyme with multiple biological activities [17]. Biochemically, PPO was characterized for both monophenolase (E.C. 1.14.18.1) and diphenolase activity (E.C. 1.10.3.1) that catalyses conversion of different monophenols to diphenols and finally, to respective quinones [17]. This enzyme is abundantly distributed in nature and possesses oxidoreductase, protease and other multiple bio-activities $[17,18]$. Owing to its phenolase activity, it is widely used in biochemical industries, industrial waste water treatment, removal of textile dyes, etc. [17]. For all these purposes, one needs a purified and immobilized enzyme that can provide the oxidoreductive activity with stability and can be reused for several times.

Till date, a number of matrices and techniques were utilized for making an immobilized PPO likewise entrapment in calcium alginate beads matrix [19], adsorption on mesoporous-activated carbons [20], chemical cross-linking on chitosan- $\mathrm{SiO}_{2}$ gel [21], etc. All these enzyme immobilization methods are indirect and these induced ways have lack of adequate stability. In this regard, the strategy of PPObased enzyme hydrogel formation provides unique dimension in developing stable smart enzyme-based biomaterials. A flexible hydrogel expected to be effective for the accommodation of enzyme within the bloated spaces of the hydrogel network that will never alter the catalytic efficiency of the enzyme. For the fulfilment of this conjecture, we have introduced our previously reported a benign supramolecular hydrogel [9]. Unlike conventional approaches, here, a smart novel enzyme hydrogel of PPO was generated through direct mixing of the benign flexible supramolecular hydrogel [9] and the PPO in an aqueous environment. Even this supramolecular hydrogel material [9] intelligently performs as potent enzyme immobilize matrix to transfigure polyphenol oxidase (10000 U) into an enzyme hydrogel, a stable immobile hydrogel-frame with enhanced redox activity than that of its pure mobile form in water medium. The luminescent signal of supramolecular hydrogel material [9] was also smartly spread into enzymehydrogel network. 


\section{Experimental}

\subsection{Materials}

All chemicals and related consumables were purchased from Sigma-Aldrich and used as received. Nuclease free

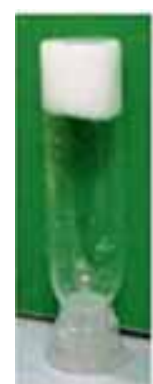

A



B
Scheme 1. Photographic presentation of enzyme hydrogel of polyphenol oxidase (10000 U). (A) Photography of supramolecular hydrogel [9] and (B) photography of enzyme hydrogel.
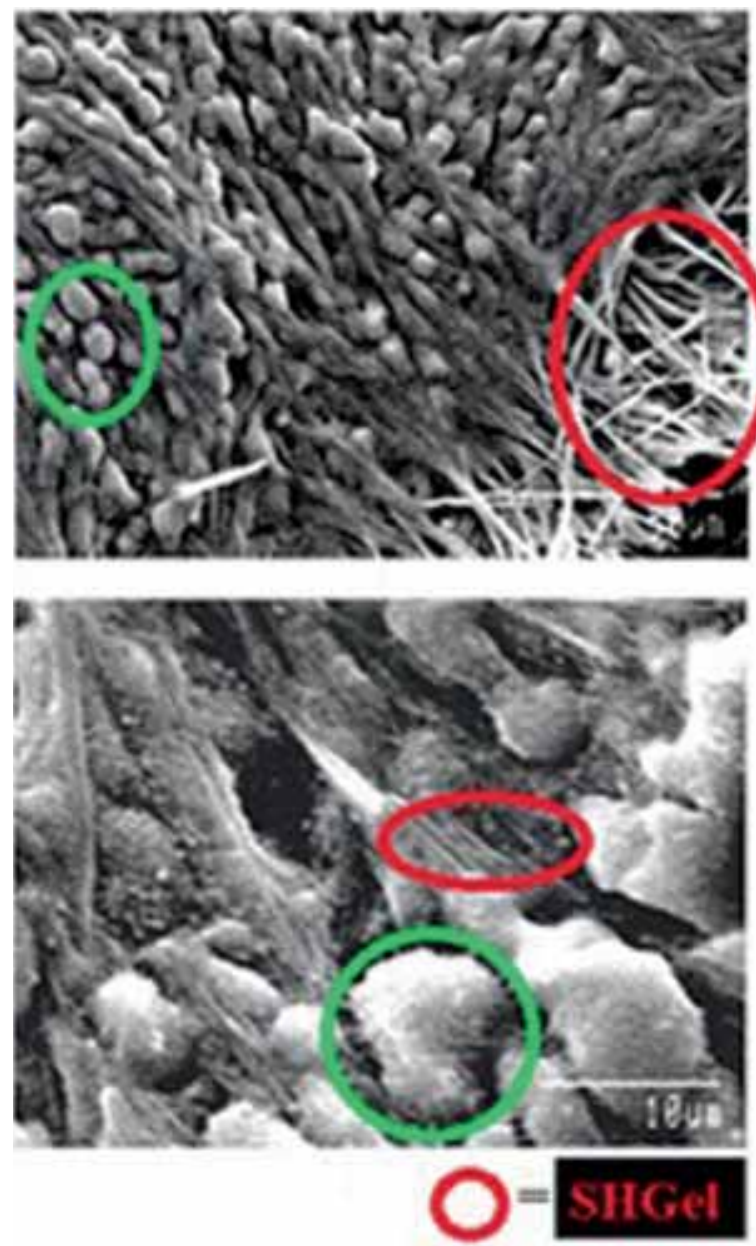

type 18 Mili-Q water was used throughout the experiment. Polyphenol oxidase enzyme (10000 U) was purchased and used as received.

\subsection{Instruments}

Absorption and fluorescence spectra were measured in a SHIMADZU UV-1800 and SHIMADZU UV-3101PC spectrophotometer and a Perkin-Elmer LS55 fluorimeter, respectively. The transmission electron microscopic (TEM) images were taken with a FEI, Tecnai $G^{2}$ F30, S-Twin microscope using an accelerating voltage of $300 \mathrm{kV}$. The TEM is also equipped with GATAN Orius CCD camera, highangle annular dark field scanning transmission electron microscopy (STEM-HAADF) mode with a HAADF detector from Fischione (Model 3000). The rheological analysis was performed using Anton Paar MCR 702 RHEOMETER with Peltier temperature control system. The samples were dispersed in deionized water by sonication and dropped onto a conventional carbon-coated copper grid. Scanning
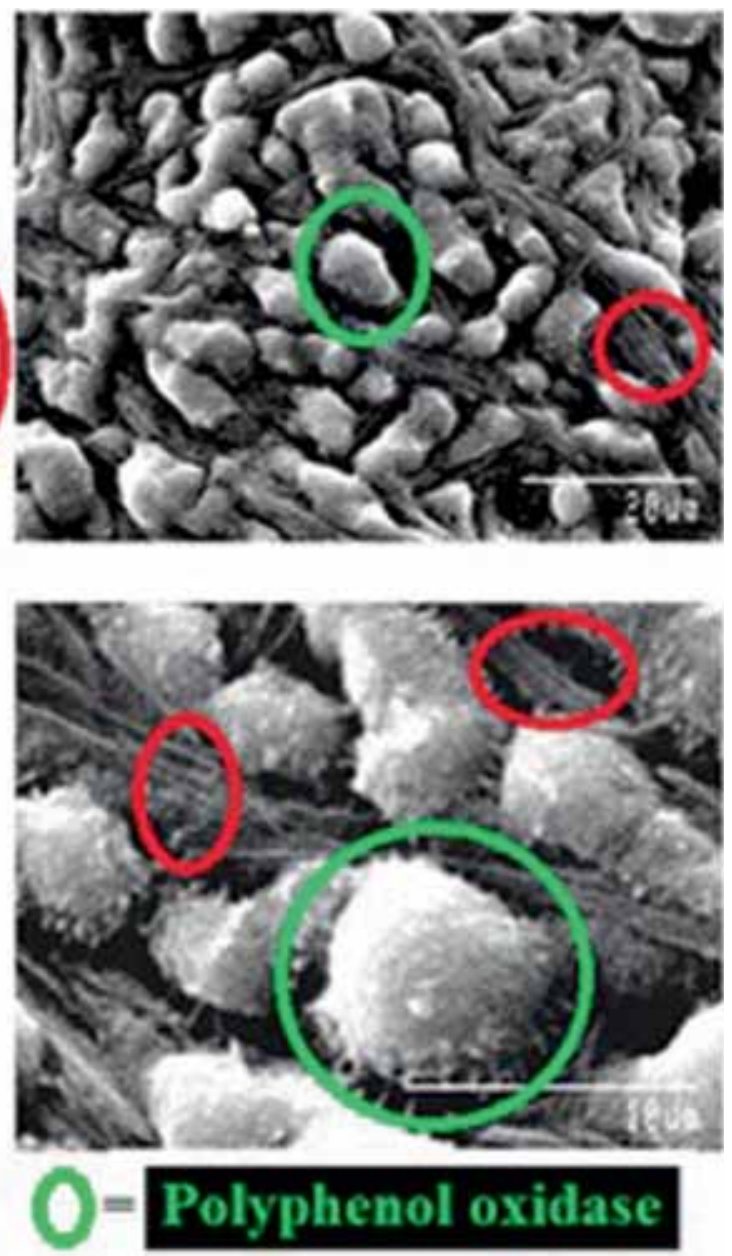

Figure 1. Microstructure of enzyme hydrogel found in scanning electron microscopic analysis. Here, SHGel = supramolecular hydrogel [9]. 
electron microscopy (SEM) images were obtained using SEM-Hitachi S-530 model with sputter gold-coater and critical point dryer. UV microscopic study was performed using Leica 224 fluorescence microscope with UV attachment.


\subsection{Synthesis of enzyme hydrogel}

The supramolecular hydrogel is prepared according to our previously published literature method [9]. The structural flexibility of the supramolecular hydrogel network [9] directs
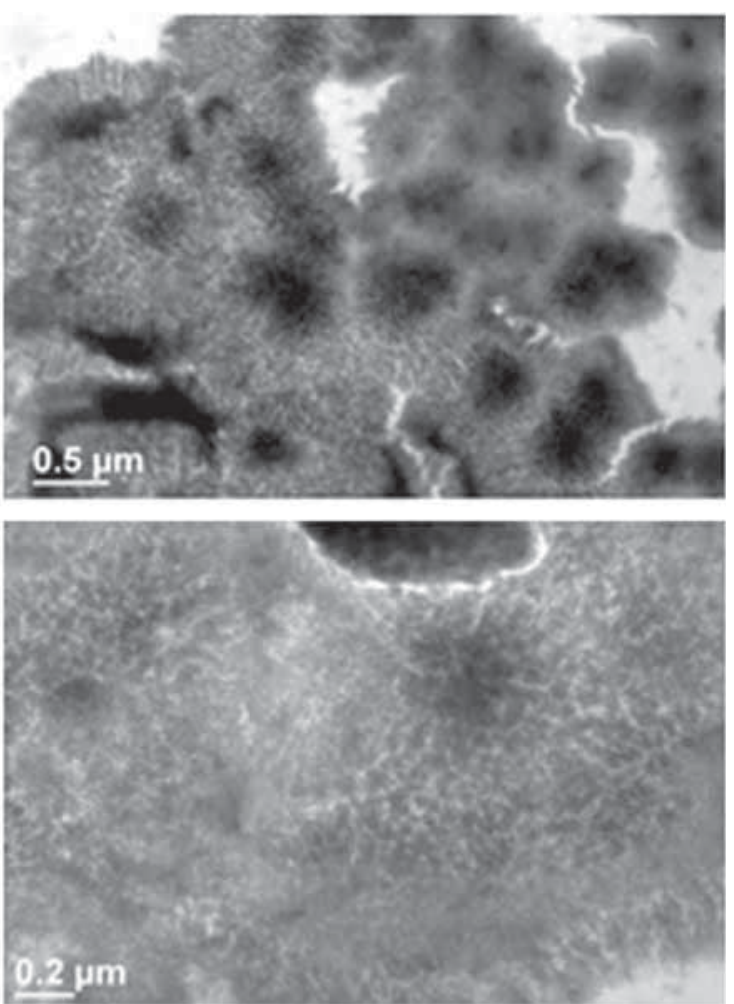

Figure 2. TEM analysis of enzyme hydrogel showing microstructure depicting the sea urchin-shaped architecture.



Figure 3. Angular frequency-dependent storage modulus $\left(G^{\prime}\right)$ and loss modulus $\left(G^{\prime \prime}\right)$ of the enzyme hydrogel. 


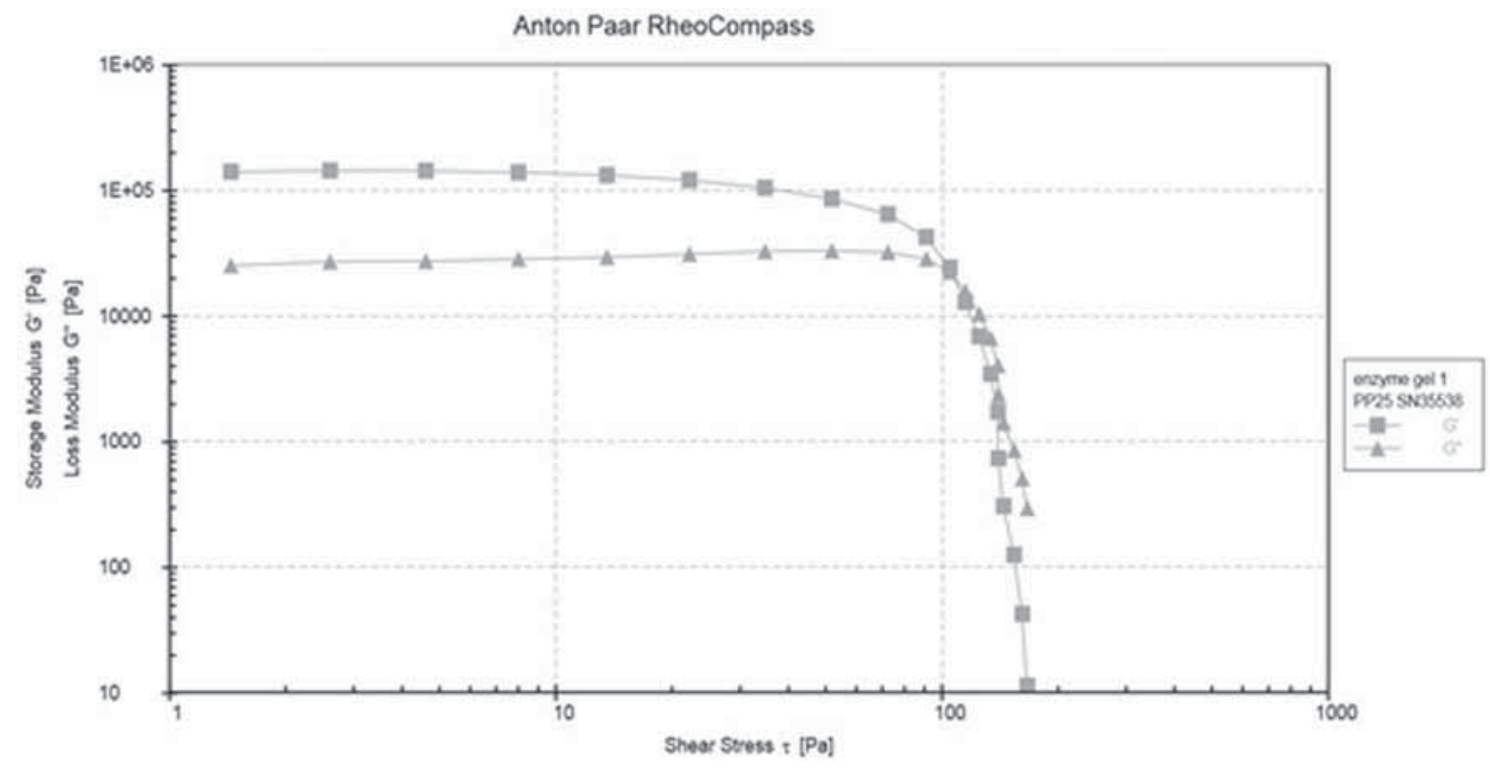

Figure 4. Plot of $\mathrm{G}^{\prime}$ and $\mathrm{G}^{\prime \prime} v$. shear stress at a constant frequency of $10 \mathrm{rad} \mathrm{s}^{-1}$ at $25^{\circ} \mathrm{C}$.

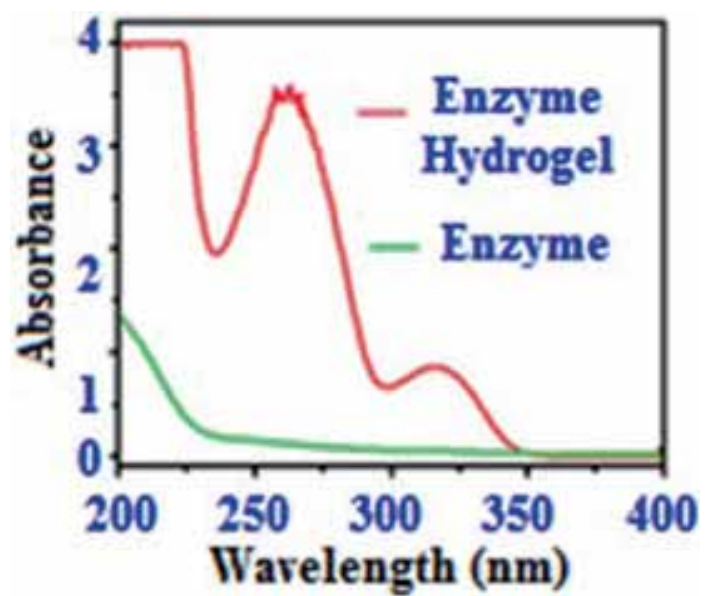

Figure 5. UV-absorption pattern of PPO enzyme and PPO-based enzyme hydrogel.

us to use it as an immobilizing scaffold for enzyme. The water solution of PPO (10000 U) is converted into an immobilizing network by forming an enzyme hydrogel through exploiting the supramolecular hydrogel material [9].

The non-toxic nature of the supramolecular hydrogel [9] is an added advantage towards the formation of another hydrogel pattern (i.e., enzyme hydrogel) with a natural enzyme without hampering its enzymatic activity. Enzyme hydrogel (scheme 1) was directly prepared in a single pot reaction through the instantaneous mixing of $100 \mu l$ aqueous solution of PPO (10000 U) into $900 \mu l$ water solution of the supramolecular hydrogel [9] (with a concentration of $100 \mu \mathrm{g} \mathrm{ml}^{-1}$ ). A transparent stable white gelatinous soft material of enzyme hydrogel was formed at room temperature under ambient conditions. Cytotoxicity of the synthesized materials was tested on rat peritoneal macrophage culture and examined through cell viability (MTT) assay as well as Giemsa's staining following our earlier report [9].

\subsection{Ethical clearance statement}

The study of cytotoxicity involving laboratory animal i.e., rat was approved by the Institutional Ethical Committee of VisvaBharati University, India (registration no. 1819/GO/Ere/S/15/ CPCSEA).

\section{Results and discussion}

\subsection{Microstructure of enzyme hydrogel}

The microstructure of this enzyme hydrogel was also characterized by SEM (figure 1). The globular micro-assemblies of PPO enzyme marked as green circle in figure 1 , are present along with fibrous supramolecular hydrogel patterns, marked as red circle in figure 1, in the PPO-based enzyme hydrogel network.

The microstructural study depicts that both enzyme molecules and supramolecular hydrogel are equally accountable for the novel enzyme hydrogel formation. Being an immobilizing scaffold, our synthesized enzyme hydrogel is an unusual approach, where PPO enzyme directly transformed into enzyme hydrogel pattern. TEM analysis (figure 2) also endorses the stability of the immobilized pattern of PPO enzyme into an enzyme-hydrogel network with its constitutional supramolecular hydrogel architecture [9]. In the TEM 
analysis, the sea-urchin-shaped architectures are due to the PPO enzyme-hydrogel molecules and the special type structural views in TEM (figure 2) clearly ratifies that PPO enzyme directly participates in the immobilizing enzyme hydrogel formation.

\subsection{Rheological analysis of enzyme hydrogel}

Generally, different supramolecular gel-like species are viscoelastic materials and these show dual characteristics including viscous and elastic properties of materials. These features are measured by the rheological analysis. Gels can store and disintegrate the energy under oscillatory strain and this is categorized through the parameters storage modulus $\left(G^{\prime}\right)$ and loss $\left(G^{\prime \prime}\right)$ modulus of the gel sample, respectively.
The storage modulus $\left(G^{\prime}\right)$ and loss modulus $\left(G^{\prime \prime}\right)$ of the samples are elaborated as [22]

$$
\begin{aligned}
G^{\prime} & =\left(\sigma_{0} / \gamma_{0}\right) \cos (\delta) . \\
G^{\prime \prime} & =\left(\sigma_{0} / \gamma_{0}\right) \sin (\delta) .
\end{aligned}
$$

The storage modulus $\left(G^{\prime}\right)$ signifies energy stored during deformation by oscillatory shear. The loss modulus $\left(G^{\prime \prime}\right)$ shows energy dissipated from the gel-like material under oscillatory shear. For a gel-like material, $G^{\prime}(\omega)>G^{\prime \prime}(\omega)$, where $\omega$ is the angular frequency.

The linear viscoelastic limit is $0.008 \%$ and the enzyme hydrogel sample was analysed within this linear viscoelastic limit, i.e., at $0.003 \%$. The frequency sweep measurement of

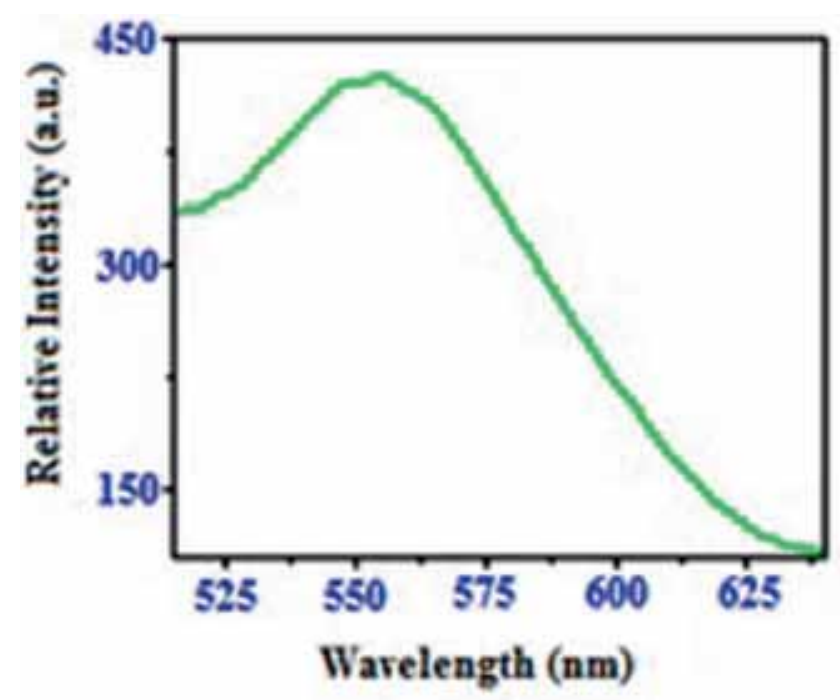

(a)



(b)

Figure 6. Luminescent feature of PPO-based enzyme hydrogel. (a) Fluorescence spectral pattern of enzyme hydrogel and (b) fluorescence micrograph of enzyme hydrogel.

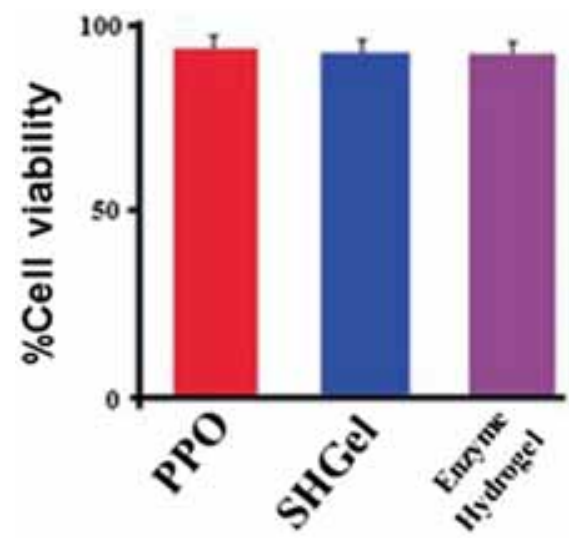

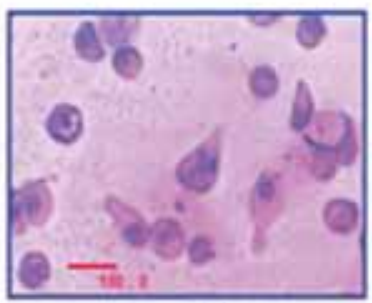

Control

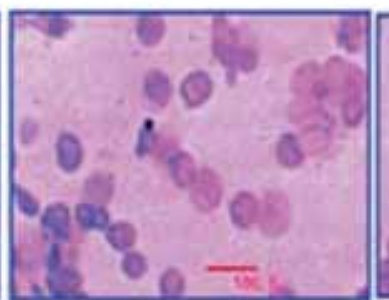

PPO



Enzyme bydrogel

Figure 7. Analysis of the toxicity of enzyme hydrogel. 
the enzyme hydrogel with a $0.003 \%$ strain is given in figure 3 . Figure 3 shows that storage modulus $\left(G^{\prime}\right)$ and loss modulus $\left(G^{\prime \prime}\right)$ were $\sim 1.54 \times 10^{5} \mathrm{~Pa}$ and $36894 \mathrm{~Pa}$, respectively. It is also found that the storage modulus is substantially greater than that of the loss modulus. It endorses the feature of a stable hydrogel network of the enzyme-based gel system. [23] Linear viscoelastic strain was quantified by strain sweep test. Linear viscoelastic limit was found to be $0.008 \%$ and yield stress was found to be $74.5 \mathrm{~Pa}$. The variations of the values of storage modulus $\left(G^{\prime}\right)$ and loss modulus $\left(G^{\prime \prime}\right)$ with shear stress at a constant frequency of $10 \mathrm{rad} \mathrm{s}^{-1}$ at $25^{\circ} \mathrm{C}$ are also given in figure 4.

\subsection{Spectral and microscopic study}

The UV-VIS absorption (figure 5) and fluorescence spectral pattern (figure 6a) of the PPO-based enzyme hydrogel was recorded. PPO-based enzyme hydrogel displays an intense broad absorption band centred at $316 \mathrm{~nm}$ and the fluorescence emission maximum at $555 \mathrm{~nm}\left(\lambda_{\mathrm{ex}}=316 \mathrm{~nm}\right)$ at $298 \mathrm{~K}$ and atmospheric pressure.

The fluorescent feature further recorded by UV-microscopy (figure 6b) demonstrates the luminescent nature of the PPObased enzyme hydrogel. Thus, the luminescent property of supramolecular hydrogel network [9] directly introduced into the PPO-based enzyme hydrogel scaffold.

\subsection{Analysis of toxicity}

Previously, we have shown non-toxic nature of supramolecular hydrogel [9]. However, in this investigation, we have also checked the toxicity of our synthesized material as chemical interaction with enzyme may lead to alteration of the cytotoxicity. We found no alteration in cell viability after



Figure 8. UV-absorption pattern for the redox efficiency of enzyme hydrogel (i.e., polyphenol oxidase (175 U)) on phenol. Here, amount of water solution of the enzyme hydrogel (with polyphenol oxidase (175 U) ) added is $10,60,160,260,300 \mu \mathrm{l}$. treating rat peritoneal macrophage culture with either PPO or enzyme hydrogel (figure 7). In addition, there was no change in the cell morphology after treatment with these materials as evident from Giemsa's staining of control and treated cells (figure 7).

\subsection{Redox activity study}

Alike PPO enzyme, this immobilized enzyme hydrogel scaffold also exerts a promising oxido-reductase activity in water medium (figures 8-11). A number of phenolic substrates involving phenol, cathechol, resorcinol and quinol are exploited to check the oxido-reductase activity of the enzyme hydrogel scaffold. We have used UV-VIS spectroscopic method for the monitoring of the enzymatic oxidation of said phenolic substrates in water medium.



Figure 9. UV-absorption pattern for the redox efficiency of enzyme hydrogel (i.e., polyphenol oxidase (175 U)) on catechol. Here, amount of water solution of the enzyme hydrogel (with polyphenol oxidase (175 U)) added is $10,60,160 \mu \mathrm{l}$.

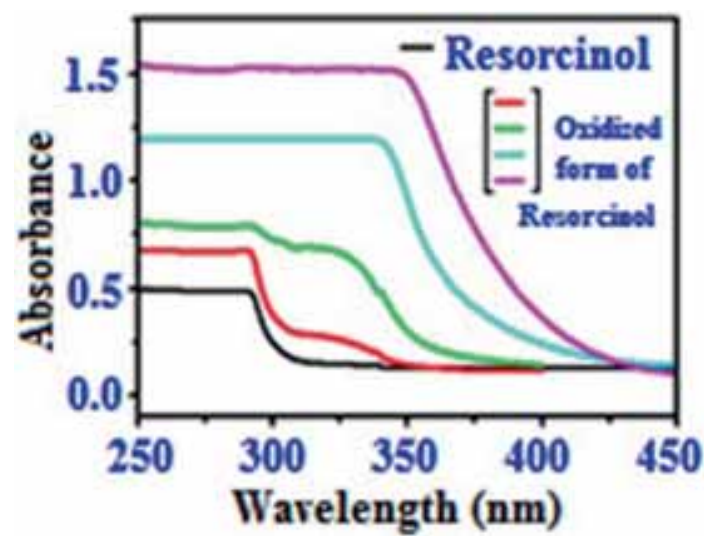

Figure 10. UV-absorption pattern for the redox efficiency of enzyme hydrogel (i.e., polyphenol oxidase (175 U)) on resorcinol. Here, amount of water solution of the enzyme hydrogel (with polyphenol oxidase (175 $\mathrm{U})$ ) added is $10,60,70,170 \mu \mathrm{l}$. 
The redox efficient luminescent PPO-based enzyme hydrogel with $175 \mathrm{U}$ polyphenol oxidase activity was progressively added into a specific volume of phenolic compounds in water medium and corresponding UV-absorption spectra (figures 8-11) was recorded for monitoring the

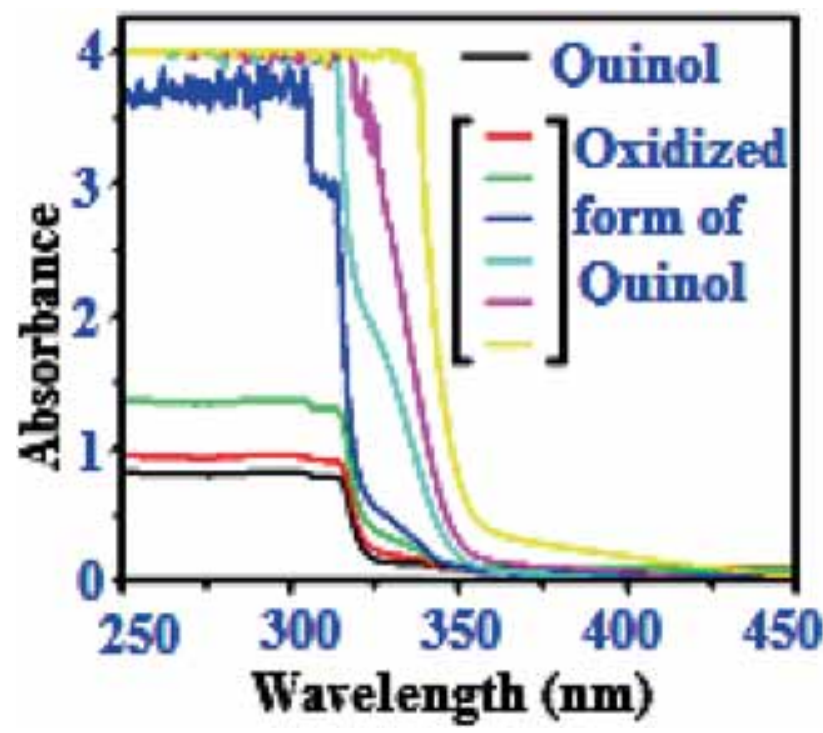

Figure 11. UV-absorption pattern for the redox efficiency of enzyme hydrogel (i.e., polyphenol oxidase (175 U)) on quinol. Here, amount of water solution of the enzyme hydrogel (with polyphenol oxidase (175 U)) added is 10, 60, 160, 260, 280, $360 \mu \mathrm{l}$. oxidation behaviour of respective phenolic compounds in water solutions (figures 8-11).

UV-absorption patterns for the redox activity of enzyme hydrogel on phenol, catechol, resorcinol and quinol are given in figures 8, 9, 10 and 11, respectively. Experimentally, the 175 U-based PPO enzyme hydrogel in different amounts were consecutively added to a fixed concentration $(1 \mathrm{mM})$ and volume $(2 \mathrm{ml})$ of each phenolic substrate, individually. UV-absorption spectroscopic study (figures 8-11) clearly indicates the enhancement of redox efficiency of this luminescent PPO-based enzyme-hydrogel network.

Unlike the enzyme hydrogel, to achieve similar extent of catalytic efficiency $355 \mathrm{U}$ soluble polyphenol oxidase was found effective. This has indicated that immobilization of polyphenol oxidase in enzyme-hydrogel network almost doubles the catalytic efficacy of the enzyme than that of its mobile form. The possible mechanistic explanation behind the better efficacy of enzyme hydrogel can be answered by the orientation of the catalytic/active site of polyphenol oxidase in hydrogel network. Enhancement of activity of any immobilized oxidoreductive enzyme is dependent on the orientation of its active site that should be in such a fashion in immobilization network that provides proximity with its substrate and facilitates the catalysis [24]. Herein, the formation of the stable enzyme hydrogel resulted from the spontaneous interaction between hydrogel and PPO which in turn provided better catalysis of the said enzyme that is in soluble form (figure 11). So, these observations alongside the microstructural fitness of the immobilized scaffold together supported



Scheme 2. The scheme depicting spontaneous transformation of PPO enzyme and SHGel in aqueous medium to yield enzyme-hydrogel. PPO possesses a catalytic site that governs the redox conversion of phenolic substrates. The better redox efficiency of PPO in enzymehydrogel is most likely due to the novel architecture of the enzyme-hydrogel scaffold, wherein mostly the catalytic sites are exposed towards the substrate. 
the postulation that catalytic site of PPO in enzyme hydrogel is most likely to be oriented in such a microenvironment that favours better interaction with the phenolic substrate for their catalytic conversion. A possible mechanism of formation of the enzyme hydrogel and its catalysis is presented in scheme 2.

\section{Conclusions}

Finally, we have prepared a non-toxic supramolecular hydrogel [9] directed PPO (10000 U)-based enzyme hydrogel. Fluorescence investigations including spectroscopic and microscopic experiments reveal the luminous feature of the enzyme hydrogel. The rheological experimental data shows that the enzyme hydrogel is a stable gel material. The enzyme hydrogel is basically an enzyme-immobilized scaffold which efficiently improves the redox activity of free PPO enzyme. The redox efficacy of the enzyme hydrogel is tested on some selective phenolic substrates like phenol, catechol, resorcinol and quinol through UV-VIS absorption spectroscopy. Moreover, the synthesized enzyme hydrogel is non-toxic, similar to our previously reported supramolecular hydrogel and the functional raw material PPO. The synthesized enzyme hydrogel is interesting not only due to the direct involvement of PPO enzyme towards hydrogel formation, but also stable, more effective and economically viable over different literature reports [19-21]. Therefore, this unique biomaterial will direct us to search its biofunctionality in living systems.

\section{Acknowledgements}

Dey is grateful to CSIR (New Delhi, India) for a research project (Project No.: 01(2866)/16/EMR-II) and for financial supports. He is also thankful to Anton Paar India Pvt. Ltd. for the unconditional help to collect rheological data.

\section{References}

[1] Kamata H, Akagi Y, Kayasuga-Kariya Y, Chung U and Sakai T 2014 Science 343873

[2] Elisseeff J 2008 Nat. Mat. 7271

[3] Babu S S, Praveen V K and Ajayaghosh A 2014 Chem. Rev. 1141973

[4] Zhao F, Ma M L and Xu B 2009 Chem. Soc. Rev. 38883

[5] Zhang J and Ma P X 2013 Adv. Drug Deliv. Rev. 651215

[6] Appel E A, Barrio J D, Loh X J and Scherman O A 2012 Chem. Soc. Rev. 416195

[7] Rybtchinski B 2011 ACS Nano 56791

[8] Singh A and Peppas N A 2014 Adv. Mater. 266530

[9] Dey B, Mondal R K, Mukherjee S, Satpati B, Mukherjee N, Mandal A et al 2015 RSC Adv. 5105961

[10] Dong R, Pang Y, Su Y and Zhu X 2015 Biomater. Sci. 3937

[11] Dey B, Mukherjee S, Mukherjee N, Mondal R K, Satpati B, Senapati D et al 2016 RSC Adv. 646573

[12] Zhu X, Ma Y, Zhao C, Lin Z, Zhang L, Chen R et al 2014 Langmuir 3015229

[13] De Hoog H-P M, Arends I W C E, Rowan A E, Cornelissen J J L M and Nolte R J M 2010 Nanoscale 2709

[14] Lee J, Ko J H, Lin E-W, Wallace P, Ruch F and Maynard H D 2015 Polym. Chem. 63443

[15] Yan J, Sun Y, Zhu H, Marcu L and Revzin A 2009 Biosens. Bioelectron. 242604

[16] Lehr J, Williamson B E, Barrière F and Downard A J 2010 Bioelectrochemistry 79142

[17] Mukherjee S, Basak B, Bhunia B, Dey A and Mondal B 2013 Rev. Environ. Sci. Biotechnol. 1261

[18] Mukherjee S, Bandyopadhayay B, Basak B, Mandal N, Dey A and Mondal B 2012 Not. Sci. Biol. 498

[19] Niladevi K N and Prema P 2008 World J. Microbiol. Biotechnol. 241215

[20] Kennedy L J, Selvi P K, Padmanabhan A, Hema K N and Sekaran G 2007 Chemosphere 69262

[21] Shao J, Ge H and Yang Y 2007 Biotechnol. Lett. 29901

[22] Yan C and Pochan D J 2010 Chem. Soc. Rev. 393528

[23] Fan H, Wang L, Feng X, Bu Y, Wu D and Jin Z 2017 Macromolecules 50666

[24] Lim S I, Yang B, Jung Y, Cha J, Cho J, Choi E S et al 2016 Sci. Rep. 639587 